\title{
Electronic active model for saccadic eye movements
}

\author{
O. Terán \& E. Suaste \\ Bioelectronics Section, Department of Electrical Engineering, \\ CINVESTAV-IPN, Mexico
}

\begin{abstract}
The design, development and construction of an electronic active model (EAM) were proposed. The model was based on the reciprocal innervation mechanical model for horizontal eye movements and was intended to emulate the biodynamic rotatory properties of a human eye plant caused by neurologic activation signals on the lateral and medial extraocular muscles. Biodynamic properties of the mechanical eye plant, such as viscosity, inertia and elasticity, were included in the EAM in order to obtain a similar response. Apart from these features, voltage controlled resistors (VCRs) based on MOSFET to keep the nonlinearity of the plant were also included. These active elements, together with passive components, constitute the structure of the extraocular muscles, eyeball and tissues around the eyeball. The activation of agonist-antagonist signals were generated by two voltage controlled sources to reproduce the neurological activity. In this way, applying the appropriate signals to generate saccadic movements was obtained by a voltage signal proportional to the velocity of the eye torque. This signal allows one to obtain information relating to acceleration and position in order to validate the EAM. Due to this capability to emulate saccadic movements, the EAM makes it possible to reproduce involuntary eye movements caused by pathologies such as Horizontal Nystagmus. Finally, some of the EAM's advantages lie in its response velocity and the ease of obtaining continuous records for the dynamic eyeball response in a signal that features ease of recording and application in biomedical areas, and is particularly relevant to specialists such as Neuro-ophthalmologists, ophthalmologists and optometrist, and even for medical education.
\end{abstract}

Keywords: eye plant, electronic model, saccadic eye movements. 


\section{Introduction}

Nowadays, models and instruments that offer valuable information regarding the visual system and make it possible to reproduce phenomenological events (generally in a particular section) are becoming more frequently found in the biomedical sciences, such as ophthalmology, neurology and physiology, which are directly linked with human vision,. One of these sections has been the oculomotor system, which is the final stage in the human control of eye movements. Descartes was the first to propose the idea of the conjugate muscles action to produce rotation of the eyeball. This principle of reciprocal innervations opens the way to new ideas that simulate the prediction of the dynamic response of eye movements [1]. A mechanical model that obtains this result was presented by Clark and Stark [2] for saccadic horizontal eye movements. Based on Descartes' principle and Hill's model to muscles [3], a mechanical model is capable of predicting magnitude, velocity and acceleration of saccadic eye movements from the primary position to a new desired position of the eye. This model has probed the efficacy of simulating the eye plant due to the considerable nonlinear properties of the extraocular eye muscles [4].

In this manner, the importance of the eye plant models lie in obtaining a new form to analyze disorders in people with some class of congenital or pathological disorder in the oculomotor system that most of the time causes a diminution in visual acuity, as occurred in the case of Nystagmus, which can only be treated to reduce it effects. For this reason, the presented design focuses on offering a tool that can be directly applied in diagnostic or research related to the human visual system. In addition, the electronic active model (EAM) is a useful tool in medicine or ophthalmologic schools for didactical purposes

\section{Method}

The sixth order model developed by Clark and Stark [4] has been studied by other authors [5-7] and the results have demonstrated that it can reproduce realistic eye position, velocity and acceleration trajectories of human saccades. For this reason, our design is based on this nonlinear mechanical model for horizontal eye movements (Fig. 1). The extraocular muscles are based on the Hill muscle model. Both contain nonlinear dashpots $\mathrm{B}_{\mathrm{AG}}$ and $\mathrm{B}_{\mathrm{AT}}$, the viscosity of which change as a function of force and velocity in the muscles. Inside each muscle, there is an active-state tension generator (ATG) responsible for producing the necessary force to the extraocular muscle contraction [8,9]. This force is the ideal physiological force generated into the muscle and cannot be measured directly. The force produced by an active state tension generator defines the magnitude and duration of saccadic eye movements; therefore it is important to control these properties. In order to simplify this control, the force produced by the ATGs is obtained from filtering a pulse-step signal with a lowpass filter, which changes its time constant in the falling edge of the signal. This is related with the activation and deactivation of cells inside the muscle [4]. 
The model element $\mathrm{J}_{\mathrm{G}}$ represents the inertia due to mass of the eye globe; $\mathrm{K}_{\mathrm{G}}$ and $\mathrm{B}_{\mathrm{G}}$ are the elasticity and viscosity caused by the tissue that surrounds the eye globe, including the lateral rectus muscles. $\mathrm{K}_{\mathrm{SE}}$ is related with the instantaneous change of length of the muscle in response to an instantaneous change of force. $\mathrm{K}_{\mathrm{P}(\mathrm{AG})}$ and $\mathrm{K}_{\mathrm{P}(\mathrm{AT})}$ are the passive elasticity of the muscle. The ATGs $\mathrm{F}_{\mathrm{AT}}$ and $\mathrm{F}_{\mathrm{AG}}$ depend directly on the pulse step signals $\mathrm{N}_{\mathrm{AG}}$ and $\mathrm{N}_{\mathrm{AT}}$. In Fig. 2 the input and output signals of the agonist and antagonist extraocular rectus muscle and the moment when the time constant change occurs in the filter are represented.

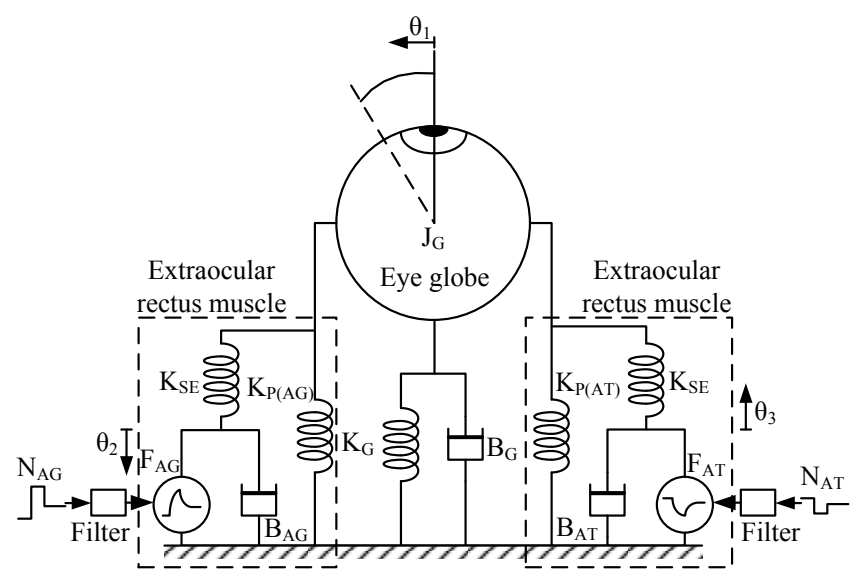

Figure 1: $\quad$ Sixth order nonlinear eye plant model for horizontal saccadic eye movements [3], $\mathrm{B}_{\mathrm{AG}}$ and $\mathrm{B}_{\mathrm{AT}}$ represent the nonlinear force-velocity relationship.

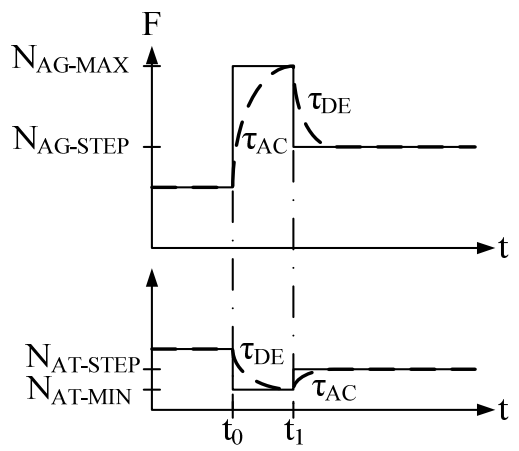

Figure 2: Controller signals transformed into active tensions by the first order filter with the two activation and deactivation constants.

The equations that govern the entire system are:

$$
F_{A G}=K_{S E}\left(\theta_{2}-\theta_{1}\right)+B_{A G} \dot{\theta}_{2}
$$




$$
\begin{gathered}
F_{A T}=K_{S E}\left(\theta_{1}-\theta_{3}\right)-B_{A T} \dot{\theta}_{3} \\
K_{S E}\left(\theta_{2}-\theta_{1}\right)-K_{S E}\left(\theta_{1}-\theta_{3}\right)=K_{P} \theta_{1}+B_{P} \dot{\theta}_{1}+J_{G} \ddot{\theta}_{1}
\end{gathered}
$$

where:

$$
\begin{gathered}
B_{A G}= \begin{cases}1.2 F_{A G} /\left(900+\dot{\theta}_{2}\right) & \dot{\theta}_{2} \geq 0 \\
3 F_{A G} / 900 & \dot{\theta}_{2}<0\end{cases} \\
B_{A T}= \begin{cases}3 F_{A T} / 900 & \dot{\theta}_{3}>0 \\
1.2 F_{A T} /\left(900+\dot{\theta}_{3}\right) & \dot{\theta}_{3} \leq 0\end{cases}
\end{gathered}
$$

In eqn. (3) the term $K_{P}$ represents the elements $K_{P(A G)}$, $K_{P(A T)}$ and $K_{G}$ that were simplified in a spring connected to the eye globe. Considering the equation of the system and the characteristics of the mechanical model, the analog circuit was obtained directly (Fig 3). According to the terminology of the mechanical model, components were named in respect to their corresponding mechanical analogies.

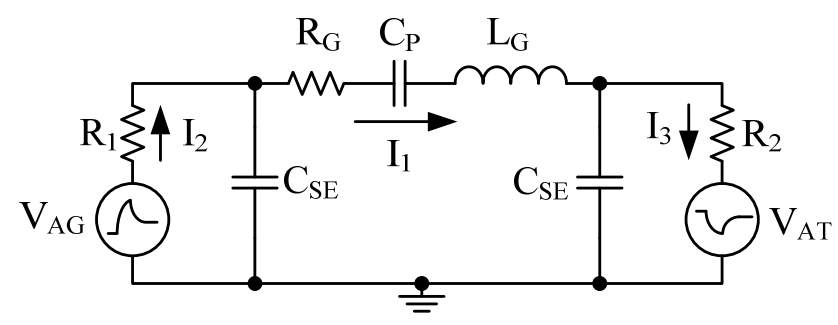

Figure 3: Simplified electronic model where $R_{1}$ and $R_{2}$ are nonlinear resistors.

The resultant circuit has three networks, which senses current on each one to represent the direction of the eye movements. As a consequence of the electrical equivalence, the magnitude of position $\theta_{1}$ is represented in the circuit by the electrical charge $q$. Therefore $I_{l}=d q / d t$ represents the velocity of the eye movements. To obtain magnitude and acceleration it is necessary to integrate and derive this current signal. The voltage sources $V_{A G}$ and $V_{A T}$ generate the equivalent tension produce by the ATGs (Fig. 2). The pulse-step signal and filter are included inside the voltage sources.

The eye plant model contains two nonlinear dashpots to satisfy the nonlinear force-velocity relationship in the muscle. In the circuit (Fig. 3) these mechanical elements are represented by nonlinear resistors $\left(R_{1}, R_{2}\right)$. Their values are given by the voltage and current in each network, as shown in the equations of the circuit. 


$$
\begin{gathered}
V_{A G}=C_{S E}^{-1} \int\left(I_{2}-I_{1}\right) d t+R_{1}\left(I_{2}\right) \\
V_{A T}=C_{S E}^{-1} \int\left(I_{1}-I_{3}\right) d t-R_{2}\left(I_{3}\right) \\
C_{S E}^{-1}\left[\int\left(I_{2}-I_{1}\right) d t-\int\left(I_{1}-I_{3}\right) d t\right]=C_{E Q}^{-1} \int I_{1} d t+R_{G} I_{1}+L_{G} \dot{I}_{1}
\end{gathered}
$$

where:

$$
\begin{aligned}
& R_{1}= \begin{cases}\frac{1.25 \cdot V_{A G}}{900+I_{2}} & I_{2} \geq 0 \\
\frac{3 \cdot V_{A G}}{900} & I_{2}<0\end{cases} \\
& R_{2}= \begin{cases}\frac{1.25 \cdot V_{A T}}{900+I_{3}} & I_{3}<0 \\
\frac{3 \cdot V_{A T}}{900} & I_{3} \geq 0\end{cases}
\end{aligned}
$$

$\mathrm{R}_{1}$ and $\mathrm{R}_{2}$ are voltage controlled resistors (VCRs) that are circuits capable of emulating an electric resistor, which have the advantage of changing resistance with a control voltage. For this reason, a VCR solves the problem of a timevariant resistor present in our EAM (Fig. 4). The VCR designed is based on the principle of resistive mirror [10]. This technique linearizes the trans-impedance response of common BJT, FET or MOSFET transistors as a function of the voltage control. To generate these voltages $\left(\mathrm{V}_{\mathrm{f} 1}=\mathrm{R}_{1}\right.$ and $\left.\mathrm{V}_{\mathrm{f} 2}=\mathrm{R}_{2}\right)$ for each VCR requires independent circuits of control $\left(f_{1}\right.$ and $\left.f_{2}\right)$ designed to response according eqns. (9) and (10). Other important blocks of the circuit are the filters, which change their time constant commuting resistors $\left(\tau_{\mathrm{AC}}, \tau_{\mathrm{DE}}\right)$ at the same time

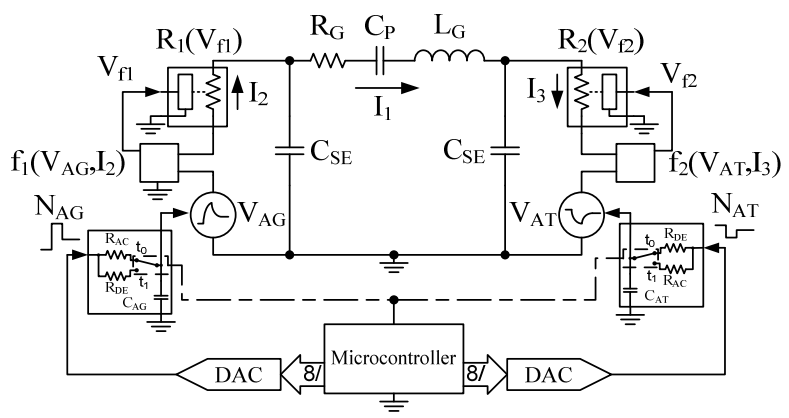

Figure 4: Block diagram of the EAM where $\mathrm{I}_{1}$ represents the eye velocity for saccadic movements. 


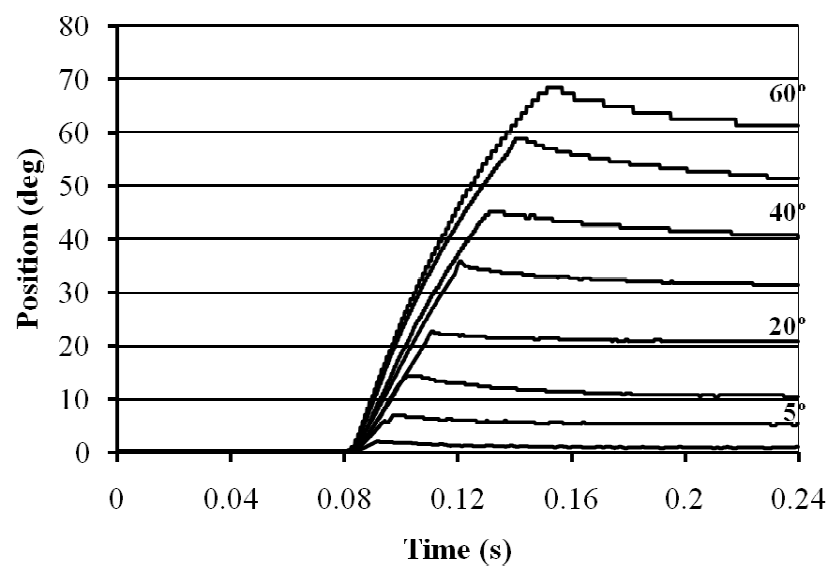

Figure 5: Position curves obtained by the EAM on $C_{P}$ for saccades with amplitudes of 1, 5, 10, 20, 30, 40, 50 and $60 \mathrm{deg}$.

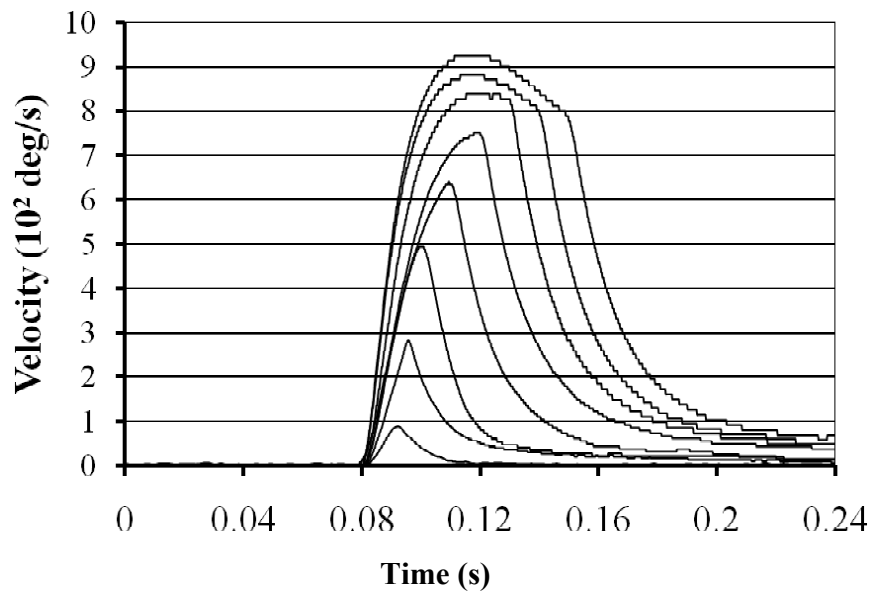

Figure 6: Velocity curves obtained by the EAM on $R_{G}$ for saccades with amplitudes of 1, 5, 10, 20, 30, 40, 50 and $60 \mathrm{deg}$.

(Fig. 2). Thus, a microcontroller coordinates the electronic switches inside the filter with the pulse-step signal $\left(\mathrm{N}_{\mathrm{AT}}, \mathrm{N}_{\mathrm{AG}}\right)$, modifying the time constants correctly. This requires that the microcontroller also controls the amplitude and duration of $\mathrm{N}_{\mathrm{AT}}$ and $\mathrm{N}_{\mathrm{AG}}$. Both parameters are easy to control digitally using a digital-analog converter for the amplitude of the signal and a timer inside the microprocessor for duration; this also guarantees the repeatability of the input stimulation voltage signal to the EAM. 


\section{Results}

The final circuit of the EAM was probed in a range of movement of 1 to 60 degrees from an initial position of 0 degrees. The final values of the electronic circuit are: $\mathrm{C}_{\mathrm{P}}=1160 \mathrm{uF}, \mathrm{R}_{\mathrm{G}}=15 \Omega, \mathrm{L}_{\mathrm{G}}=47 \mathrm{mH}, \mathrm{C}_{\mathrm{SE}}=560 \mathrm{uF}, \mathrm{R}_{\mathrm{AC}}=1.8 \mathrm{k} \Omega$, $\mathrm{R}_{\mathrm{DE}}=3.6 \mathrm{k} \Omega, \mathrm{C}_{\mathrm{AG}}=2.2 \mathrm{uF}$ and $\mathrm{C}_{\mathrm{ANT}}=2.2 \mathrm{uF}$. All of these values were obtained from the original mechanical model multiplied by a factor of 1000 to simplify the design. The electronic ATGs are related to the mechanical force in a scale of $1 \mathrm{gf}=0.01$ V. Position (Fig. 5), velocity (Fig. 6) and acceleration (Fig. 7) curves were obtained through voltage on $\mathrm{C}_{\mathrm{P}}, \mathrm{R}_{\mathrm{G}}$ and $\mathrm{L}_{\mathrm{G}}$, respectively, taking advantage of the capability of these components to derivate $\left(\mathrm{L}_{\mathrm{G}}\right)$, integrate $\left(\mathrm{C}_{\mathrm{P}}\right)$ or leave the wave form of the current without change $\left(\mathrm{R}_{\mathrm{P}}\right)$. However, it is necessary for $\mathrm{L}_{\mathrm{G}}$ to have a high quality factor in order to minimize the error caused by electric resistance of the wire.

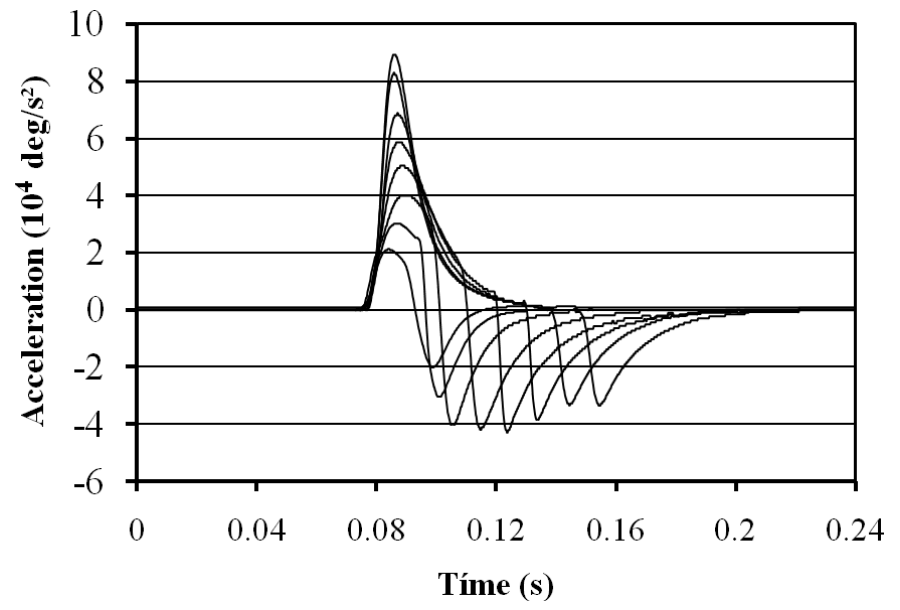

Figure 7: Acceleration curves obtained by the $\mathrm{EAM}$ on $\mathrm{L}_{\mathrm{G}}$ for saccades with amplitudes of 1, 5, 10, 20, 30, 40, 50 and $60 \mathrm{deg}$.

To obtain the appropriate magnitude scale, position voltages need to be multiplied by a factor of $C_{P}, R_{G}$ for velocities and $1 / L_{G}$ for acceleration voltage curves and each one multiplied by 100000 to compensate for the reduction in the voltage of the ATGs and increments in the values of the components in the circuit.

In order to evaluate the goodness of the EAM results, the voltage curves were analyzed according with the main-sequence diagrams for human eye movements [1] and compared (Fig. 8) with saccade eye movement signals obtained by high velocity video-oculography [11], the curve positions and the velocity obtained at the maximum deviation of $\pm 10 \mathrm{~ms}$ for the duration of the saccades, \pm 3 deg from the desired position, $+100 \mathrm{deg} / \mathrm{s}$. Many of these EAM curves do not match with the experimental curves due to biological variations that modify the dynamical responses of the human eye plant. 


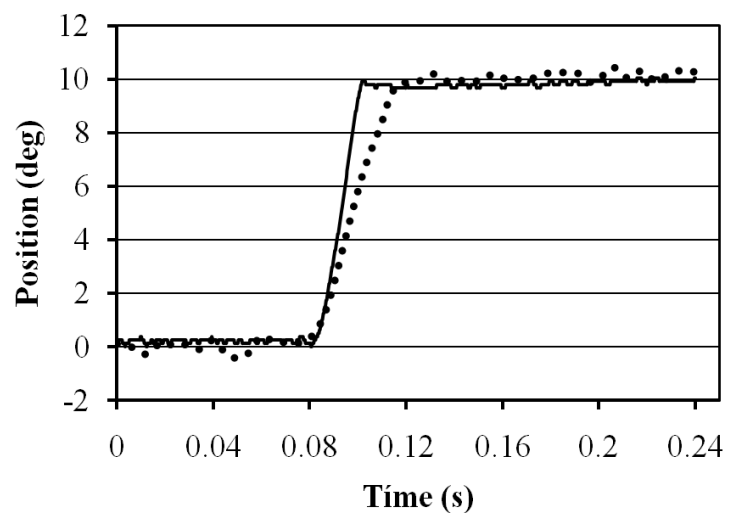

a)

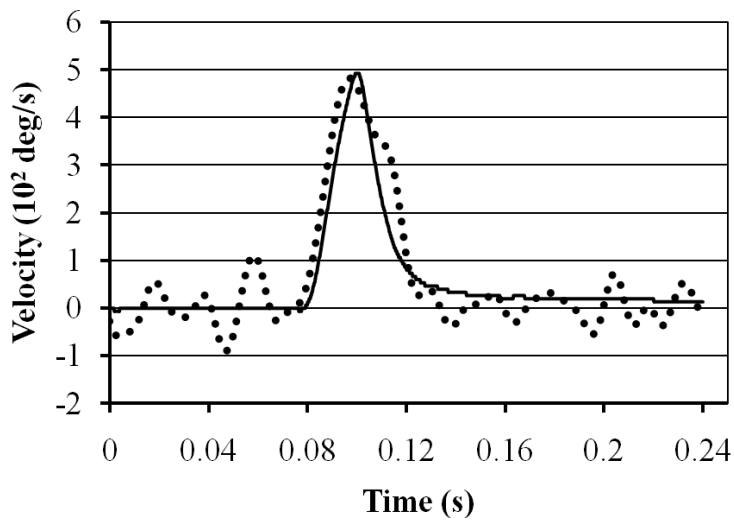

b)

Figure 8: a) The solid line is the postion estimation generated by the EAM, the dotted line is the position trajectory obtained by high velocity video-oculography, both curves for a $10 \mathrm{deg}$ saccade. b) The solid line is the velocity estimation directly generated by the EAM, the dotted line is a curve velocity obtained from the derived position curve in a), both curves were generated from a 10 deg saccade.

\section{Discussion}

The designed EAM is capable of emulating the saccadic eye movements as suggested by the comparison with experimental results. According with experimental researches by several authors [1, 5, 6, 12-14], $10^{\circ}$ saccades generated by EAM comply with $50^{\circ} \mathrm{ms}$ of duration, peak velocity of $500 \mathrm{deg} / \mathrm{s}$ and acceleration of $40000 \mathrm{deg} / \mathrm{s}^{2}$. The EAM has been based on nonlinear models complying with the nonlinear force-velocity relationship present in extra ocular eye muscles. A VCR was designed with the experimental response in the 
required resistive range and with the greater linearity in the saccadic signals bandwidth [15]. The resistive range of the VCR was determined by the experimental probes in an arrangement of saccade amplitude of 1 to 60 degrees.

Due to the amplitude of the saccadic eye movement signals obtained by the EAM, these do not need to be conditioned to be measured by an instrument, such as an oscilloscope, except where the application has a great demand for current. The EAM may function completely without the necessity to use a computer because the activation state signals are easily generated and manipulated with the aid of a microcontroller or another source can be used to generate the desired stimulation signals.

\section{References}

[1] Bahill, A. T., Bioengineering: Biomedical, Medical and Clinical Engineering, Prentice-Hall, Inc, Englewood Cliffs, New Jersey, pp. 112200, 1981, 1981.

[2] Clark, M. R. \& Stark, L., Control of Human Eye Movements: III. Dynamic Characteristics of the Eye Tracking Mechanism. Mathematical Biosciences, 20, pp. 239-265, 1974

[3] Clark, M. R. \& Stark, L., Control of Human Eye Movements: I. Modeling of Extraocular Muscle. Mathematical Biosciences, 20, pp. 191-211, 1974.

[4] Clark, M. R. \& Stark, L., Control of Human Eye Movements: II. A Model for the Extraocular Plant Mechanism. Mathematical Biosciences, 20, pp. 213-238, 1974.

[5] Enderle, J. D., Wolfe, J. W., Time Optimal Control of Saccadic Eye Movements. IEEE Transactions on Biomedical Engineering, 34(1), pp. 4355, 1987.

[6] Bahil, A. T., Latimer, J. R., Troost, B. T., Linear Homeomorphic Model for Human Movement. IEEE Transactions on Biomedical Engineering, 27(11), pp. 631-639, 1980.

[7] Martin, C. F., Schovanec, L., Muscle Mechanics and Dynamics of Ocular Motion. Journal of Mathematical Systems, Estimation and Control, 8(2), pp. 1-15, 1998.

[8] Robinson, D. A., The Mechanics of human Saccadic Eye Movement. $J$. Physiol., 174, pp. 245-264, 1964.

[9] Enderle, J. D., Bronzino, J. D., Blanchard, S. M., Physiological Modeling (Chapter 12). Introduction to Biomedical Engineering, Academic Press, pp. 693-797, 2005.

[10] Tadic, N., Resistive Mirror-Based Voltage controlled Resistor with Generalized Active Devices. IEEE Transactions on Instrumentation and Measurement, 42(2), pp. 587-591, 1998.

[11] Villamar L A and Suaste E (2008) High Velocity Videoculography to Determination of the Pupil Dynamics, American Institute of Physics Conf. Proc. Vol. 1032, Tenth Symposium on Med. Phys., Melville, New York, pp. 276-279, 2008. 
[12] Enderle, J. D., Wolfe J. W. \& Yates J. T., The Linear Homeomorphic Saccadic Eye Movement Model-A Modification. IEEE Transactions on Biomedical Engineering, 31(11), pp. 717-720, 1984.

[13] Clark, M. R., Stark, L., Time Optimal of Human Saccadic Eye Movements. IEEE Transactions on Automation Control, 20(3), pp. 345-348, 1975.

[14] Pfann, K. D., Keller, E. L. \& Miller, J. M., New Models of the Oculomotor Mechanics Based on Data Obtained with Chronic Muscle Force Transducers. Annals of Biomedical Engineering, 23, pp. 346-358, 1995

[15] Zuber, B. L., Semmlow, J. L. \& Stark, L., Frequency characteristics of the Saccadic Eye Movement. Biophysical Journal, 8(11), pp. 1288-1298, 1986. 